\title{
Structural enzymology at the legume-microbe interface: S-adenosyl-L-homocysteine hydrolase of rhizobia
}

\author{
TOMASZ MANSZEWSKI ${ }^{1}$, KRITI SINGH ${ }^{1}$, BARBARA IMIOLCZYK ${ }^{1}$, MARIUSZ JASKOLSKI ${ }^{1,2 *}$ \\ ${ }^{1}$ Institute of Bioorganic Chemistry, Polish Academy of Sciences, Poznań, Poland \\ ${ }^{2}$ Faculty of Chemistry, Adam Mickiewicz University, Poznań, Poland \\ *Corresponding author: mariuszj@amu.edu.pl
}

\begin{abstract}
S-adenosyl-L-methionine (SAM) is the most common substrate used in biological methylation reactions. During methyl group transfer, S-adenosyl-L-homocysteine (SAH) is formed as a byproduct. Since SAH is a strong inhibitor of the ubiquitous SAM dependent methylation reactions, removal of SAH by enzymatic hydrolysis serves as an important mechanism in the control of cellular methylation processes.
\end{abstract}

\section{Introduction}

S-adenosyl-L-homocysteine hydrolase (SAHase) catalyzes the hydrolysis of SAH to adenosine and L-homocysteine in a reversible reaction (de la Haba et al., 1959). The equilibrium of the hydrolysis reaction is shifted far in the direction of the substrate synthesis but under physiological conditions the products are quickly removed by other enzymatic processes (Richards et al., 1978). The enzymatic process catalyzed by SAHases is quite complex and involves a redox step. This is why the enzyme requires $\mathrm{NAD}^{+}$as a cofactor for its enzymatic competence.

Crystal structures of several SAHases are known, including enzymes from mammals, protozoans and bacteria. The enzymes are typically homotetrameric, with each subunit comprised of two principal domains: a substrate binding domain and a cofactor $\left(\mathrm{NAD}^{+}\right)$binding domain. Phylogenetic and biochemical analyses indicate that some groups of SAHases may be significantly different. In particular, plant SAHases have a unique sequence signature and are believed to function as dimers $(\mathrm{Gu}-$ ranowski et. al., 1977; Brzezinski et al., 2008).

In this project, we focus on the crystal structure of SAHases from legume plants, such as yellow lupine (Brzezinski et al., 2012), and from their nitrogen-fixing bacterial symbionts (rhizobia), looking for possible interdependencies during the formation and existence of the symbiotic association. Here, we present the results of our crystallographic analysis of recombinant SAHase

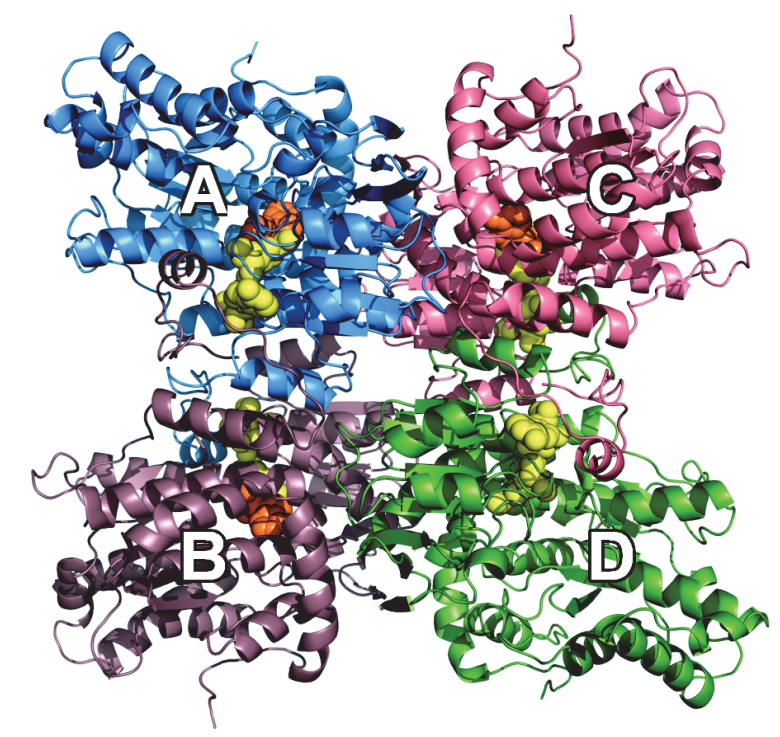

Fig. 1. A ribbon diagram of the BeSAHase tetramer, with subunits labeled A, B, C, D. NAD ${ }^{+}$and adenosine molecules are in space-filling representation

from Bradyrhizobium elkanii (BeSAHase), which infects soybean. It is the first structure of this enzyme from a bacterium capable of assimilating atmospheric nitrogen.

\section{BeSAHase in complex with adenosine molecules}

Single crystals of BeSAHase were grown by vapor diffusion in hanging drops using protein preparations preincubated with the $\mathrm{NAD}^{+}$cofactor. They diffracted synchrotron radiation to $1.7 \AA$. The structure was solved by molecular replacement, using as a model the struc- 


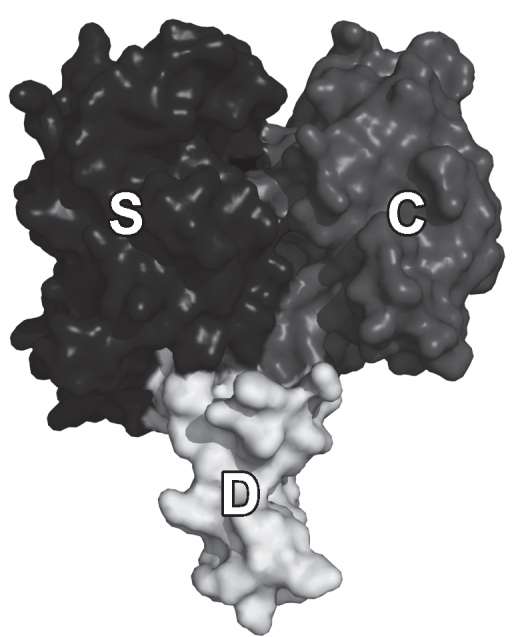

Fig. 2a. A subunit of BeSAHase in closed conformation

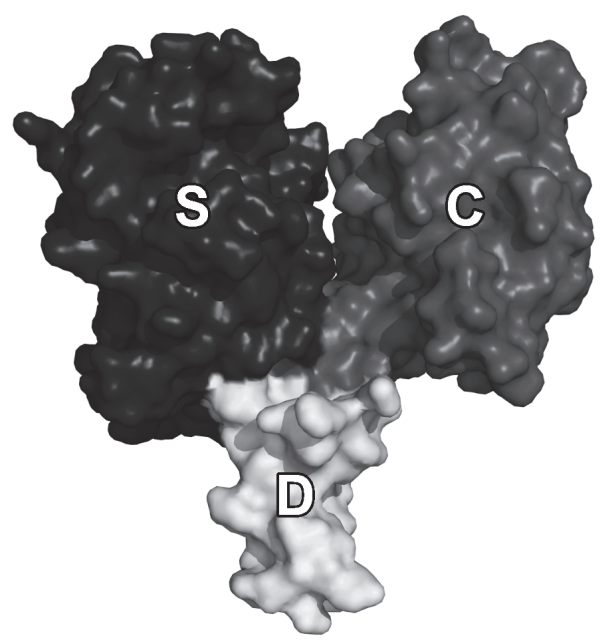

Fig. 2b. A subunit of BeSAHase in open conformation. The domains, shown in shades of gray, are labeled S (substrate-binding domain), $\mathrm{C}$ (cofactor binding domain), and $\mathrm{D}$ (C-terminal dimerization domain)

ture of SAHase from B. melitensis, and refined to $\mathrm{R} / \mathrm{R}_{\text {free }}$ of $0.1496 / 0.1757$. The crystals are orthorhombic, space group $P 2_{1} 22$, with four protein molecules in the asymmetric unit (473 amino acid residues each) forming a tetrameric enzyme (Fig. 1). There is one cofactor molecule bound in each subunit, as required by the enzymatic mechanism. In addition, a molecule of adenosine (Ado) is present in three subunits. In consequence, the homotetrameric enzyme is found in a mixed conformational state: while the Adobinding subunits are in a closed conformation, the fourth subunit is open (Fig. 2). The structure gives us a unique glimpse of SAHase during a conformational transformation required for its enzymatic cycle.

\section{BeSAHase in complexes with other ligand molecules}

The protein was also studied after preincubation with 3-deoxyadenosine (cordycepin). The crystals have the same $P 2{ }_{1} 2{ }_{1} 2$ symmetry and diffracted X-rays to a $1.84 \AA$ resolution. The cordycepin molecule was found in only one out of the four subunits. The remaining three subunits are occupied with adenosine ligands.

To obtain BeSAHase crystals with uniform ligand occupation, we elaborated a new purification protocol that includes an extra step designed to wash out all of the bound ligand molecules first, according to a modified procedure of Yuan et al. (1993). Following this procedure, a complex with 2 '-deoxyadenosine was crystallized and its crystal structure was solved to $1.54 \AA$ resolution, also in space group $P 2{ }_{1} 2_{1} 2$. The structure is currently being refined.

\section{Other biophysical studies and the future}

The protein and its complexes have also been characterized by several biophysical methods, including Dynamic Light Scattering (DLS), Small-Angle X-ray Scattering (SAXS), Isothermal Titration Calorimetry (ITC), Mass Spectroscopy (MS) and Thermofluor analysis. The results are currently being analyzed. Our crystallographic observations of this tetrameric protein in complexes with different ligands and in different binding modes are used to map the geometrical transformations connected with the complicated chemistry catalysed by this enzyme.

\section{Acknowledgments}

The project was sponsored in part by funds from the European Union within the European Regional Development Fund.

\section{References}

Brzezinski K., Bujacz G., Jaskolski M. (2008) Acta Cryst. F64: 671-673.

Brzezinski K., Dauter Z., Jaskolski M. (2012) Acta Cryst. D68: 218-231.

De La Haba G., Cantoni G. L. (1959) J. Biol. Chem. 234: 603608.

Guranowski A., Pawełkiewicz J. (1977) Eur. J. Biochem. 80: 517-523.

Richards H.H., Chiang P.K., Cantoni G.L. (1978) J. Biol. Chem. 253: 4476-4480.

Yuan C.S., Yeh J., Liu S., Borchardt R.T. (1993) J. Biol. Chem. 268: 17030-17037. 